

Journal of Computational Interdisciplinary Sciences (2009) 1(2): 149-157

(C) 2009 Pan-American Association of Computational Interdisciplinary Sciences

ISSN 1983-8409

http://epacis.org

\title{
Investigation of static curve information for multiobjective system identification
}

\author{
Samir Angelo Milani Martins ${ }^{1}$, Daniel Cunha de Souza Braga ${ }^{2}$, Erivelton Geraldo Nepomuceno ${ }^{3}$, \\ Thiago Veloso Gomes ${ }^{4}$ and Maria Luiza Figueiredo Reis ${ }^{5}$
}

Manuscript received on November 2, 2008 / accepted on January 12, 2009

\begin{abstract}
System Identification is an area of knowledge that aims at finding models by means of observations. Dynamic input-output data are usually collected in order to select structure and estimate parameters of an specific mathematical representation. In this case, a mono-objective optimization is employed, as the Least Square Method, in order to maximize the prediction accuracy. More recently, multiobjective optimization has been used for system identification, taking in account auxiliary information, such as fixed points and static curve, which is usually used to increase the global validity. It is stated in literature that there is a tradeoff between global validity and prediction accuracy (Aguirre et al., IEEE Trans. Circuits Syst., 40, 1081-1085, 2000). In this paper, it is shown that the tradeoff between objectives in a multiobjective system identification depends on the decision criterion. In fact, using the Euclidean norm of a bi-objective system identification in two real systems, as the amount of auxiliary information increases, the prediction accuracy behaves irregularly. This suggests an increment of importance for criterion decision in a MSI.
\end{abstract}

Keywords: Multiobjective System Identification, auxiliary information, static curve.

Correspondence to: Erivelton Geraldo Nepomuceno

Electrical Engineering Department, Federal University of São João del-Rei, 36307-352 São João del-Rei, MG, Brazil.

${ }^{1}$ E-mail: samir_bsmg@hotmail.com

2E-mail: eng.daniel.cunha@gmail.com

3E-mail: nepomuceno@ufsj.edu.br

4E-mail: veloso.gomes@yahoo.com.br

5E-mail: malu_cax@yahoo.com.br 


\section{INTRODUCTION}

System Identification (SI) is an old and relevant field of science. It is an area of knowledge that studies ways of modeling and system analysis (Ljung 1987; Billings 1980). A model is defined as the set of hypothesis on the structure or behavior of a physical system. From the mathematical point of view, a model is an abstraction of a real system expressed by means of equations. Models can be found in Engineering (Chiras et al. 2001; Deane and Hamill 1991), Biology (Murray 1993) among other areas of science.

System Identification usually is divided into three categories: black-box, white-box and gray-box. Black-box identification uses input and output data, usually acquired from dynamical tests, as the only source of information about the system (Nepomuceno et al. 2003; Lindskog and Sjoberg 1995). In such a framework, other features of the system are not directly considered, as the static function of a system, which describe the relation of input and output data in a steady-state regime. The black-box identification presents, as advantage, an ability to fit models based only in input and output data. However, sometimes these models do not represent the whole dynamic of the system. White-box identification obtains models from physical aspects of the process. The white-box models are usually very complete, but, they are really complex and presents difficulty to be applied, manipulated and analyzed. As a combination of qualities from white and black-box identification, the gray-box identification aims at finding models by means of real data, incorporating some features of the physical process, which is usually called auxiliary information or a priori knowledge (Aguirre et al. 2000; Abonyi et al. 2000; Petrick and Wigdorowitz 1997; Pearson and Pottmann 2000; Nepomuceno et al. 2007).

One of the main stages of the $\mathrm{SI}$ is the parameter estimation. In general, a mono-objective approach is used to achieve this task (Johansen and Babuska 2003). This means that the optimization mechanism must minimize a functional (a function whose image is scalar). However, this approach shows a disadvantage of taking only one objective into account. To overcome this problem, a technique of SI has been investigated that looks for incorporating auxiliary information in the model. This information is expressed by means of another functional, that must be minimized in set with the existing functionals. This is called as Multiobjective System Identification (MSI) (Nepomuceno et al. 2007; Aguirre et al. 2000) ${ }^{1}$.
There are many types of mathematical representation of dynamics systems such as Neural Networks (Lindskog and Sjoberg 1995), Fuzzy Logic (Takagi and Sugeno 1985), Volterra's representation, NARMAX (Nonlinear AutoRegressive Moving Average model with eXogenous input) models and others. This paper is concerned in polynomial NARMAX models representation with the use of auxiliary information, in particular the static curve. The frequent use of models and applications in nonlinear systems suggests the importance of the incorporation of auxiliary information in a model to increase the prediction accuracy. Characteristic of the static curve of the systems in different operation points has been used as auxiliary information in several works (Nepomuceno et al. 2007; Nepomuceno et al. 2004; Correa et al. 2002; Abonyi et al. 2000; Aguirre et al. 2000).

More recently, multiobjective optimization has been used for system identification, taking in account auxiliary information, which is usually used to increase the global validity. The literature suggests that there is a tradeoff between global validity and prediction accuracy (Aguirre et al. 2000). This affirmation, however, does not take into account which decision criterion is used. This contradiction is investigated in this paper. The remainder of the paper is as follows. Section 2 presents some preliminary concepts about system identification. The methodology is shown in Section 3. After that, the results are presented and in Section 5, the final remarks and future research are discussed.

\section{PRELIMINARY CONCEPTS}

In this section, preliminary concepts are presented. First, a brief overview of system identification is presented. After that, a general description of NARMAX models and multiobjective system identification is presented. Finally, two real systems used as test-system are described. The systems are: i) a DC-DC Buck Converter (Aguirre et al. 2000), which static curve was obtained by means of knowledge of the process; and ii) Electrical Heater, which real data was collected to describe its static curve (Aguirre et al. 2004).

\subsection{System Identification}

Generally, system identification may be conducted following five steps.

1. Dynamical tests and data acquisition;

Data are collected by means of static or dynamical acquisition. Static acquisition is performed in the permanent

\footnotetext{
${ }^{1}$ A series of works in literature prefers the denomination Gray-box Identification (Lorito 1998; Jorgensen \& Hangos 1995) rather than Multiobjective System Identification. However, the Multiobjective nomenclature offers a more adequate meaning for the use of auxiliary information from the author's point of view.
} 
regime, whereas the dynamical acquisition is performed during the dynamical behavior of the system.

2. Choice of mathematical representation

In this work, only NARMAX models are considered. The choice of the mathematical representation is a very difficult task and it is not considered in this work. Further information about this issue may be obtained in works as (Letellier et al. 2007; Pearson 2003; Aguirre 1997; Petrick and Wigdorowitz 1997; Aguirre and Mendes 1996; Aguirre and Billings 1995).

3. Determination of structure

There are many techniques to select structure. In this work, the structure are selected using the Error Reduction Ratio (ERR) and Akaike Information Criteria (AIC) (Aguirre and Billings 1995; Aguirre 1994).

\section{Parameter Estimation}

Parameter Estimation quantifies the contribution of each regressors in the whole model. During this procedure, the multiobjective approach is undertaken.

\section{Validation}

In this stage, the RMSE (Root Mean Square Error) index was generally used to compare and state if a model is suitable or not. Equation (1) defines this index.

$$
\operatorname{RMSE}=\frac{\sqrt{\sum_{k=1}^{N}(y(k)-\hat{y}(k))^{2}}}{\sqrt{\sum_{k=1}^{N}(y(k)-\bar{y})^{2}}},
$$

where $\hat{y}(k)$ is the $n$ step ahead prediction, $\bar{y}$ is the mean of output, $y(k)$ and $N$ is the number of samples of the validation data.

\subsection{NARMAX models}

NARMAX models (Billings 1980) describe nonlinear systems in terms of difference equations relating the current output to combinations of past inputs and outputs. It is used for control problems, where the main goal is to achieve a simple system description.

In particular, the polynomial NARMAX model can be represented as:

$$
\begin{gathered}
y(k)=F^{\ell}\left[y(k-1), \cdots, y\left(k-n_{y}\right), u(k-1), \cdots,\right. \\
\left.u\left(k-n_{u}\right), e(k-1), \cdots, e\left(k-n_{e}\right)\right]+\Xi(k),
\end{gathered}
$$

where $y(k)$ is the output, $u(k)$ is the exogenous input, and $e(k)$ is the noise signal. $\Xi(k)$ represents the prediction error. $n_{y}, n_{u}$, and $n_{e}$ are the auto-regressive, exogenous input and moving average orders, respectively. The function $F^{\ell}$ may represent a great variety of functions, including linear functions. In this paper, $F^{\ell}$ is restricted to nonlinear polynomial functions and $e(k)$ is not considered.

\subsection{Mono and Multiobjective System Identification}

In the Mono-objective Identification, it is only taken in account one objective for the estimation of the parameters, generally the prediction error. Using a deterministic method to minimize the cost function that represents the prediction error (Least Square Method - LSM), the parameters are estimated by the following way:

$$
\hat{\theta}=\left[\psi^{T} \psi\right]^{-1} \psi^{T} y
$$

where $y$ is the data vector of the temporal series, $\psi^{T}$ contains the linear and nonlinear combinations of the terms of input and output of the model, including the instant $(k-1), \hat{\theta}$ is the vector of estimated parameters.

In the Multiobjective Optimization, the cost function is not a scalar function, but a vectorial function. A flowchart of MSI is shown in Figure 1.

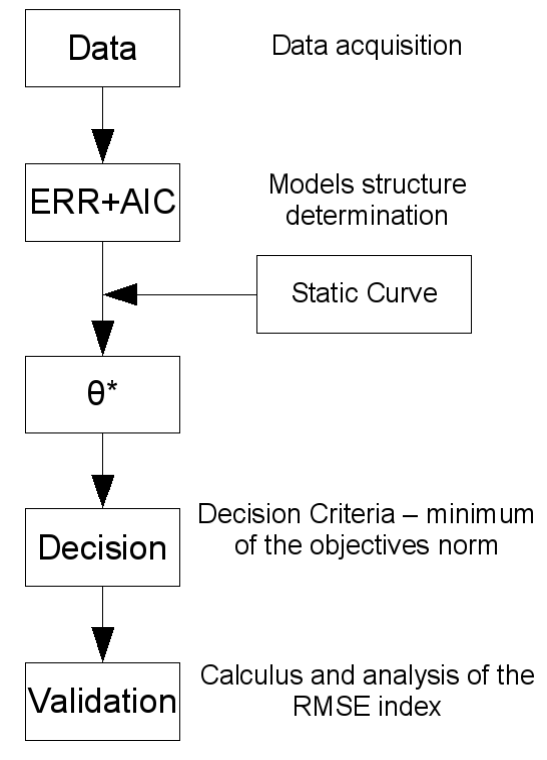

Figure 1 - Flowchart of Multiobjective Identification.

In the MSI developed in (Nepomuceno et al. 2007), any kind of information about the system can be incorporated, since that it can be written in form of prediction error. Using a extended method of the LSM, the parameters are obtained by:

$$
\hat{\theta}^{*}=\left[\sum_{i=1}^{m} w_{i} G_{i}{ }^{T} G_{i}\right]^{-1}\left[\sum_{i=1}^{m} w_{i} G_{i}{ }^{T} V_{i}\right]
$$


where $\hat{\theta}^{*}$ is parameters vector to be obtained, $G_{i}$ is information concerning the system, $V_{i}$ represents the data that has this information a priori and $m$ is the number of objectives in question (Nepomuceno et al. 2007).

This multiobjective approach yields a set of models, usually called Pareto-set. The best model from the Pareto-set is chosen following a decision criterion (Takahashi et al. 1997). In this paper, the decision criterion is based on the Euclidean norm of the objectives. Thus, the model that minimizes the Euclidean norm of objectives is chosen as best one. Figure 2 presents some Euclidean norms of distinct models. The model $N_{3}$ minimizes the Euclidean norm.

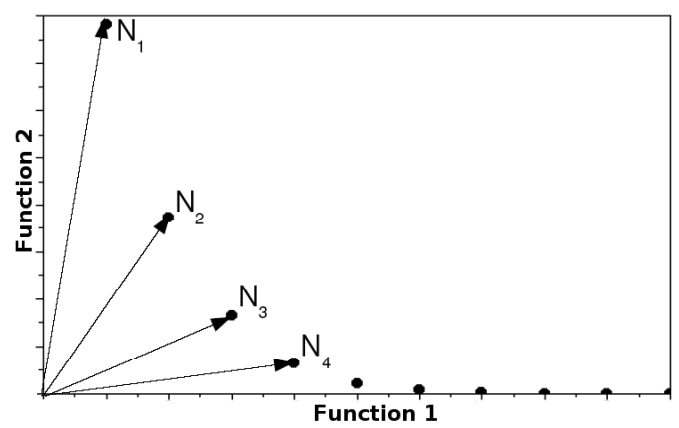

Figure 2 - Euclidean norm of points of a hypothetical Pareto-set.



Figure 3 - Static curve collected of the Electrical Heater.

\subsection{DC-DC Buck Converter and Electrical Heater}

A power electronic system known as DC-DC Buck Converter and a Electrical Heater were used as test-systems. The DC-DC Buck is a converter which consists on the voltage regulation of the load, that can be controlled by a MOSFET IRF840 (Fig. 4). The duty cycle is defined as the reason where the converter finds on for the total time of operation. When close to the unitary value, the current through the inductor and the voltage across the load increases, therefore the source energizes the loop formed for it, the capacitor and the inductor. However, when the duty cycle tends to zero, the voltage decreases with a different dynamic behavior, characterizing the nonlinearity of the system (Correa et al. 2002; Aguirre et al. 2000; Nepomuceno et al. 2007). The Electrical Heater (Fig. 5) was build with set of fans that can be on or off. Thus, the dissipation can be varied. The temperature of the Electrical Heater is measured by a temperature sensor. The input signal is the electrical power delivered and the output signal is the temperature. Both signals are in per unit system pu. For this system, 1 pu of the input signal corresponds to 136 Volts RMS and $1 \mathrm{pu}$ of the output signal is $998.51^{\circ}$ Celsius.

\section{METHODOLOGY}

The structure of the polynomial models are the same as that used in (Nepomuceno et al. 2007) and (Correa et al. 2002) for DC-DC Buck and Electrical Heater, respectively. Equation (6) presents the structure for DC-DC Buck and Equation (5) presents the structure for Electrical Heater.

$$
\begin{gathered}
y(k)=\theta_{1} y(k-1)+\theta_{2} y(k-2)+\theta_{3}+\theta_{4} u(k-1) \\
+\theta_{5} u(k-1)^{2}+\theta_{6} u(k-2) u(k-1) \\
+\theta_{7} u(k-2)+\theta_{8} u(k-2)^{2}+\xi(k) \\
y(k)=\theta_{1} y(k-1)+\theta_{2} u(k-2) u(k-1) \\
+\theta_{3} u(k-1)^{2}+\theta_{4} y(k-2) \\
+\theta_{5} u(k-2) y(k-1)+\theta_{6} u(k-2) y(k-2) \\
+\theta_{7} u(k-2)^{2}+\xi(k)
\end{gathered}
$$

$y(k)$ is the system output forecast by the model in the instant $k$, $u(k)$ is the input of the system, $\theta_{i}, i=1,2, \cdots, 8$ are the parameters to be estimated using both approaches and $\xi$ is the residues, used only during the parameter estimation.

The estimation of parameters of the model in mono and multiobjective approach incorporates static curve of the DC-DC Buck Converter and static curve of the Electrical Heater, as auxiliary information. For the DC-DC Buck Converter, the relation between input voltage and output voltage is given by a function, expressed by:

$$
v_{0}=\frac{4 v_{d}}{3}-\frac{v_{d}}{3} u,
$$

where $v_{d}$ is the constant voltage of $24 \mathrm{~V}, v_{0}$ is the value of the output voltage, in permanent regime, and $u$ is the voltage in the load controlled by the transistor IRF840 (Aguirre et al. 2000), according to Figure 4. 


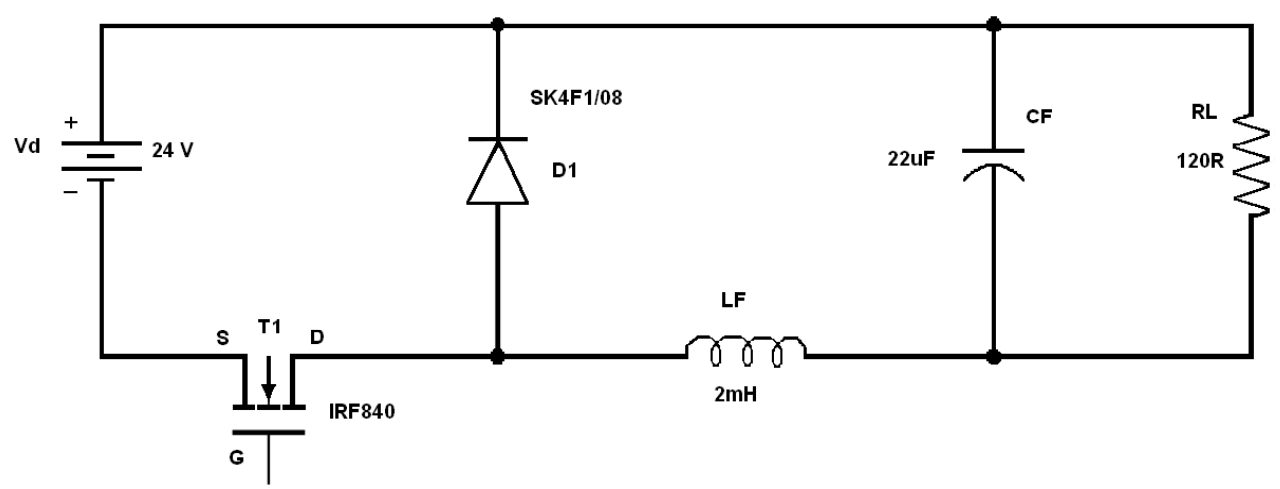

Figure 4 - DC-DC Buck Converter.

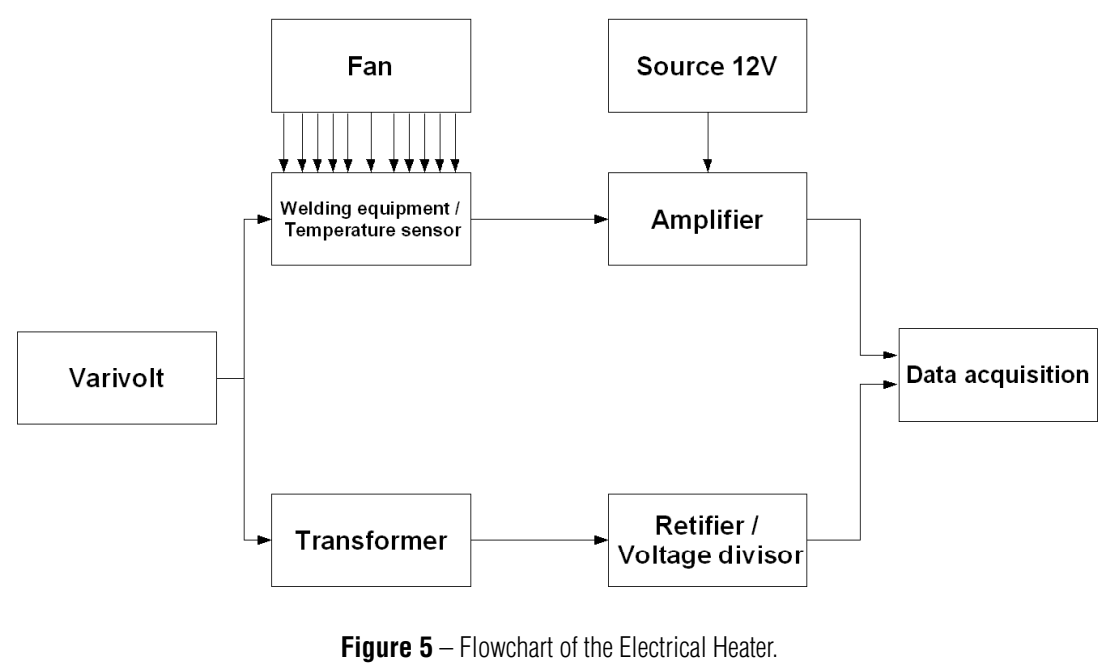

For the Electrical Heater, the static curve were collected during the permanent regime of the system presented in Figure 3. The real dynamical data of the systems are presented in Figures 7 and 8 . The static data of the Electrical Heater is shown in Figure 3.

In order to investigate the influence of static curve, simulations were carried out where one point of the static curve (auxiliary information) were added each time. On the first simulation was incorporated the central point of static curve such as Figure 6(a). On the second simulation, it was incorporated in addition of the first point (in blue), one second point (in red at Fig. 6(b)) and on the third simulation was incorporated one third point such as Figure 6(c). Other points are added in the same way. Comparatively, this simulation may be viewed as opening window from the central point to start and end points. This procedure guarantees an incremental use of auxiliary information. For each set of points, a MSI is undertaken and a Pareto-set is obtained. From each Pareto-set a model that minimizes the Euclidean norm of objectives is chosen. The same procedure was made for the DC-DC
Buck Converter and for the Electrical Heater. It was obtained 16 models for the Electrical Heater and 50 (fifty) models for the DCDC Buck Converter, in the range of $0 \mathrm{~V}$ to $4 \mathrm{~V}$. A mono-objective model also was obtained for both systems, for comparison.

\section{RESULTS}

The RMSE index are presented in Table 1 for the mono-objectives models and for each best Multiobjective model. The RMSE index for the Multiobjective models are the best Multiobjective models obtained. For the Buck converter, it is obtained when 50 points were incorporated, whereas for the Electrical Heater the best Multiobjective model was obtained in six situations, when 5, 7, 9, 11,13 and 15 points are used.

Table 1 - Best RMSE index for the models.

\begin{tabular}{|c|c|c|}
\hline & DC-DC Buck Converter & Electrical Heater \\
\hline Mono-objective & 0.1563776 & 0.3418174 \\
\hline Multiobjective & 0.1558556 & 0.3418174 \\
\hline
\end{tabular}




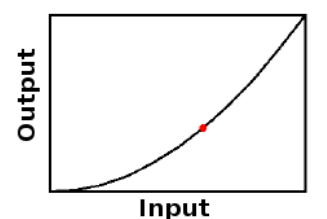

(a)



(b)

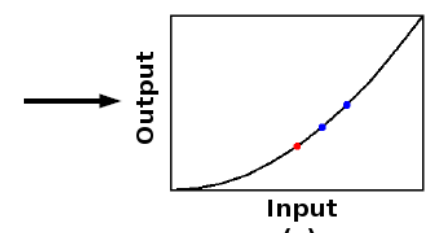

(c)

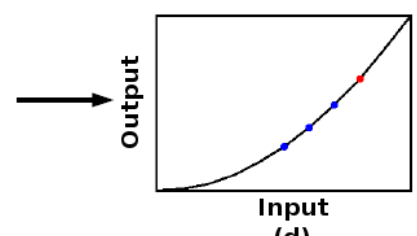

(d)

Figure 6 - Opening Window Simulation.


Figure 7 - Real data collected of a DC-DC Buck Converter.
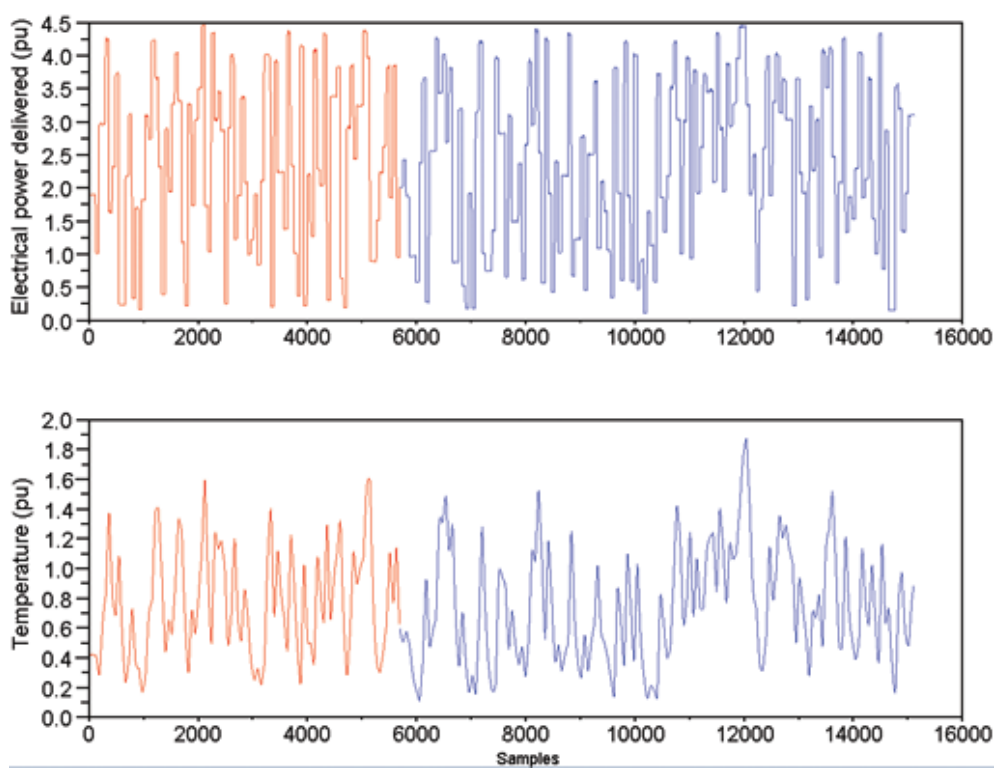

Figure 8 - Real data collected of a Electrical Heater. 
Figures 9 and 10 present the evolution of the RMSE index when was incorporated all available auxiliary information. Onestep-ahead prediction is shown in Figures 11 and 13 for the DCDC Buck Converter and in Figures 12 and 14 for the Electrical Heater.



Figure 9 - Evolution of the RMSE index for the DC-DC Buck Converter.



Figure 10 - Evolution of the RMSE index for the Electrical Heater.

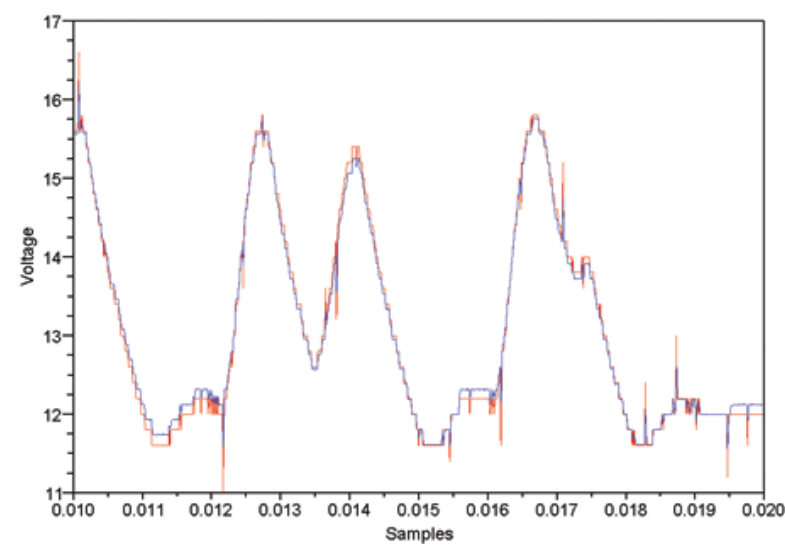

Figure 11 - Mono-objective Identification of a DC-DC Buck Converter.



Figure 12 - Mono-objective Identification of the Electrical Heater.

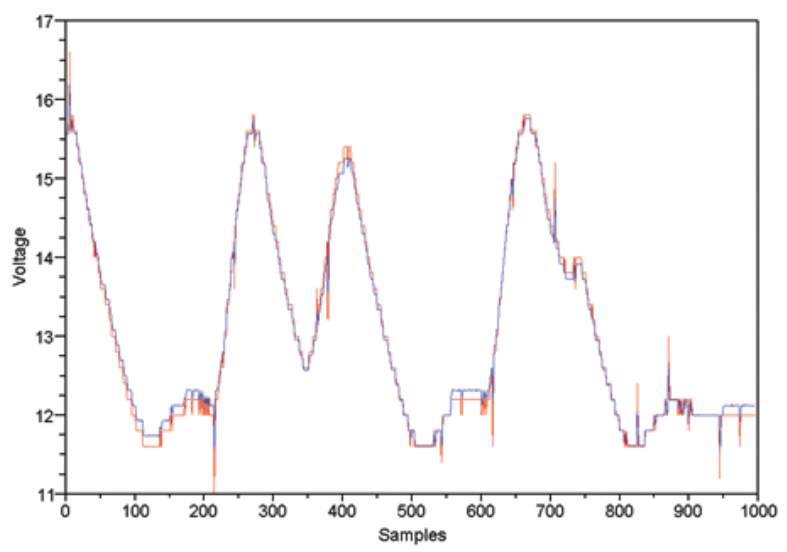

Figure 13 - MSI of the DC-DC Buck Converter for the static curve.



Figure 14 - MSI of the Electrical Heater for the static curve.

It is possible to observe by means of Table 1 , that the RMSE index of the Electrical Heater was the same for the Mono-objective and Multiobjective approach. However, the Multiobjective model incorporate the static characteristics of the model.

Under a decision criterion previous established (minimum of the Euclidean norm), the increase of the incorporation of statics characteristics in the model does not guarantee an improvement of RMSE index. This is shown in the Figures 9 and 10. However, 
for the case of DC-DC Buck Converter, the incorporation of this information makes the RMSE index from the Multiobjective approach inferior than RMSE index from the Mono-objective approach. Over there, the Multiobjective model has the static characteristics of the original system.

The RMSE index from the Electrical Heater presents a more irregular than DC-DC Buck Converter. This behavior may be explained by bias during static data acquisition of the Electrical Heater. For the DC-DC Buck Converter the static data was obtained by means of physical knowledge of process.

\section{CONCLUSIONS}

In this paper, it was verified that it is possible to increase the quality of NARMAX polynomial models when auxiliary information is incorporated in the parameter estimation. It was used real data of a DC-DC Buck Converter and an Electrical Heater, dynamics systems that presents nonlinearity. The characteristic of the static curve of this converter was used as a priori information, in different points of operation as static data (based on the power electronics theory), whereas for the Electrical Heater, the static curve are collected from a real system.

It was investigated the RMSE behavior when it is incorporated statics characteristics over different ranges. For this purpose, it was applied the Multiobjective and Mono-objective approach. For the MSI, it was incorporated static curve in a increasing way. It was shown that an increase in the amount of static curve information in the parameter estimation does not necessarily cause a improvement of the RMSE index, under a decision criteria previous established. Multiobjective models also are able of to present others systems characteristics, like static characteristics, and others. Over there, the MSI presents an inferior RMSE for the systems in question. This suggests an increment of importance for criterion decision in a MSI.

Future research may incorporate other types of auxiliary information. It is also desired, the investigation of auxiliary information for structure selection of NARMAX models.

\section{ACKNOWLEDGMENTS}

The authors are very grateful to PIIC/UFSJ for the support.

\section{REFERENCES}

ABONYI J, BABUSKA R, VERBRUGGEN HB \& SZEIFERT F. 2000. Incorporating prior knowledge in fuzzy model identification. International Journal of Systems Science, 31(5): 657-667.
AGUIRRE LA. 1994. Some remarks on structure selection for nonlinear models. International Journal of Bifurcation and Chaos, 4: 1707-1714.

AGUIRRE LA. 1997. On the structure of nonlinear polynomial models: higher order correlation functions, spectra, and term clusters. IEEE Transactions on Circuits and Systems Part I: Fundamental Theory and Applications, 44(5): 450-453.

AGUIRRE LA \& BILLINGS SA. 1995. Improved structure selection for nonlinear models based on term clustering. International Journal of Control 62(3): 569-587.

AGUIRRE LA, DONOSO-GARCIA PF \& SANTOS R. 2000. Use of $a$ priori information in the identification of global nonlinear models - A case study using a buck converter. IEEE Transactions on Circuits and Systems I - Fundamental Theory and Applications, 47(7): 1081-1085.

AGUIRRE LA \& MENDES EMAM. 1996. Global nonlinear polynomial models: structure, term cluster and fixed points. International Journal of Bifurcation and Chaos, 6(2): 279-294.

AGUIRRE L, BARROSO M, SALDANHA R \& MENDES E. 2004. Imposing steady-state performance on identified nonlinear polynomial models by means of constrained parameter estimation. IEE Proceedings, Part D: Control Theory and Applications, 151(2): 174-179.

BILLINGS SA. 1980. Identification of nonlinear systems - a survey. IEE Proceedings-D Control Theory and Applications, 127(6): 272-285.

CHIRAS N, EVANS C \& REES D. 2001. Nonlinear gas turbine modeling using NARMAX structures. IEEE Transactions on Instrumentation and Measurement, 50(4): 893-898.

CORREA MV, AGUIRRE LA \& SALDANHA RR. 2002. Using steady-state prior knowledge to constrain parameter estimates in nonlinear system identification. IEEE Transactions on Circuits and Systems I - Fundamental Theory and Applications, 49(9): 1376-1381.

DEANE JHB \& HAMILL DC. 1991. Chaotic behavior in current-mode controlled dc-dc converter. Electronics Letters, 27(13): 1172-1173.

JOHANSEN TA \& BABUSKA R. 2003. Multi-objective identification of Takagi-Sugeno fuzzy models. IEEE Transactions on Fuzzy Systems, 11(6): 847-860.

JORGENSEN SB \& HANGOS KM. 1995. Grey box modelling for control: qualitative models as a unifying framework. International Journal of Adaptative Control and Signal Processing, 9(6): 547-562.

LETELLIER C, AMARAL GFV \& AGUIRRE LA. 2007. Insights into the algebraic structure of lorenz-like systems using feedback circuit analysis and piecewise affine models. Chaos An Interdisciplinary Jr of Nonlinear Science, 17(2): 1-11.

LINDSKOG P \& SJOBERG J. 1995. A comparison between semiphysical and black-box neural net modeling: a case study, in BULSARI 
AB \& KALLIO S. (eds). Proceedings of the International Conference: Engineering Applications of Artificial Neural Networks, Helsinki, Finland, pp. 235-238.

LJUNG L. 1987. System Identification: Theory for the User. PrenticeHall, London.

LORITO F. 1998. Identification of a grey-box model of nonlinear current transformer for simulations purposes. Control Engineering Practice, 6: 1331-1339.

MURRAY JD. 1993. Mathematical Biology, Berlin: Springer.

NEPOMUCENO EG, NETO OM, TAKAHASHI RHC \& TAVARES CE. 2003. A heuristic approach to robust control design for power systems with several facts devices. International Journal of Electrical Power and Energy Systems, 25(1): 13-20.

NEPOMUCENO EG, TAKAHASHI RHC \& AGUIRRE LA. 2007. Multiobjective parameter estimation for non-linear systems: affine information and least-squares formulation. International Journal of Control, 80(6): 863-871.
NEPOMUCENO EG, TAKAHASHI RHC, AGUIRRE LA, NETO OM \& MENDES EMAM. 2004. Multiobjective nonlinear system identification: a case study with thyristor controlled series capacitor (TCSC). International Journal of Systems Science 35(9): 537-546.

PEARSON RK. 2003. Selecting nonlinear model structures for computer control. Journal of Process Control, 13: 1-26.

PEARSON RK \& POTTMANN M. 2000. Gray-box identification of blockoriented nonlinear models. Journal of Process Control, 10: 301-315.

PETRICK MH \& WIGDOROWITZ B. 1997. A priori nonlinear model structure selection for system identification. Control Engineering Practice, 5(8): 1053-1062.

TAKAGI T \& SUGENO M. 1985. Fuzzy identification of systems and its applications to modeling and control. IEEE Transactions on Systems Man and Cybernetics 15(1): 116-132.

TAKAHASHI RHC, PERES PLD \& FERREIRA PAV. 1997. Multiobjective $\mathrm{H}-2 / \mathrm{H}$ infinity guaranteed cost PID design. IEEE Control Systems Magazine, 17(5): 37-47. 